This page is intentionally left blank 


\section{IN THE INTERMISSIONS...}

\section{Collected Works on Research into the}

Essentials of Theoretical Physics in Russian Federal Nuclear Center,

Arzamas-16

\section{Editor}

\section{Yu. A. Trutnev}

Russian Federal Nuclear Center 
Published by

World Scientific Publishing Co. Pte. Ltd.

P O Box 128, Farrer Road, Singapore 912805

USA office: Suite 1B, 1060 Main Street, River Edge, NJ 07661

UK office: 57 Shelton Street, Covent Garden, London WC2H 9HE

\section{British Library Cataloguing-in-Publication Data}

A catalogue record for this book is available from the British Library.

\section{IN THE INTERMISSIONS ...}

Copyright $(1998$ by World Scientific Publishing Co. Pte. Ltd.

All rights reserved. This book, or parts thereof, may not be reproduced in any form or by any means, electronic or mechanical, including photocopying, recording or any information storage and retrieval system now known or to be invented, without written permission from the Publisher.

For photocopying of material in this volume, please pay a copying fee through the Copyright Clearance Center, Inc., 222 Rosewood Drive, Danvers, MA 01923, USA. In this case permission to photocopy is not required from the publisher.

ISBN 981-02-3606-9

This book is printed on acid-free paper. 


\section{FOREWORD}

A fragment of a letter written by Yu. B. Khariton, the Scientific Leader of RFNC from its foundation in 1946 to 1993, is facsimiled. This letter supporting the idea of publication of the present volume turned out to be the last document in his life.

...The Russian Federal Nuclear Center (RFNC) - All-Russia Scientific Research Institute of Experimental Physics was founded 50 years ago for the development of the nuclear weapons like Los Alamos National Laboratory in the U.S.A. Over extended period, it was a top secret Institute; the closed town Arzamas-16 (presently Sarov) where it is located was shown on no accessible map. In the last few years, the secret cover essentially thawed. The world has became aware of the detailed history of the advent of the Soviet nuclear weapons. It transpires that the scientists of RFNC were engaged not only in weapons investigations. It was just here that the ideas of the magnetic confinement of plasma, laser-driven fusion, pulsed high magnetic field were originally conceived, and besides RFNC pioneered some other works important in physical application.

Meanwhile the fact that a lot of highest-level results in the essentials of theoretical physics were obtained here remains almost unknown. This is partly because the papers (with supporting fictitious affiliations or even with no address) were published in Soviet physical journals many of which did not attract the proper attention in the West or were not translated into European languages altogether.

The establishment of the Arzamas school of theoretical physics and its creative style owe their origins to the "superstars" of the Soviet physics and mathematics, such as N. N. Bogoliubov, N. A. Dmitriev, D. A. Frank-Kamenetskiǔ, M. A. Lavrent'ev, L. V. Ovsyannikov, I. Ya. Pomeranchuk, A. D. Sakharov (Nobel Prize winner of 1975), I. E. Tamm (Nobel Prize winner of 1958), V. S. Vladimirov, Ya. B. Zel'dovich. A number of eminent physicsresearchers, such as A. I. Baz', V. L. Ginzburg, I. M. Khalatnikov, L. D. Landau (Nobel Prize winner of 1962), worked closely with the RFNC theorists and exerted an appreciable effect on the formation of the Arzamas school of theoretical physics although they were never staff members of the Institute.

Many results of the studies carried out at the Institute were not coming to light until that some former weapons scientists taking positions in academical institutions wrote and published their monographs which eventually gained general acceptance. It is appropriate at this point to recall the world-wide renowned monographs by N. N. Bogoliubov and D. V. Shirkov, Introduction to 
the Theory of Quantized Fields (Interscience, New York 1959), D. Frank-Kamenetskiı̌, Physical Processes in Stellar Interiors (Israel Program for Scientific Translations, Jerusalem, 1962), L. V. Ovsyannikov, Group Analysis of Differential Equations (Academic, New York 1982), Ya. B. Zel'dovich and Yu. P. Raizer, Physics of Shock Waves and High-Temperature Hydrodynamic Phenomena (Academic, New York 1966).

A number of scientists of the Institute endeavour presently to upheld the reputation of the Arzamas theoretical school and are actively pursuing investigations into fundamental areas of theoretical physics. The publication of the 'Collection' will give impetus to these activities, provide opportunities for weapons scientists to redirect their talents to other scientific areas, promote exchanging the scientific experience and establishing new bonds with international academical centers.

The papers of the 'Collection' contain pioneer results some of which are yet of basic importance in the present state of the art even though had been obtained in the past.

The issue will undoubtedly be beneficial to anyone active or interested in fundamentals of theoretical physics, from professional theorists to graduate students, it will also perform a useful service for experts in history and methodology of physics.

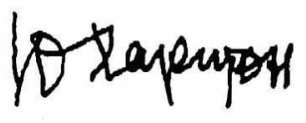

Yu. B. Khariton

RFNC Scientific Leader Emeritus Full Member of the Russian Academy of Sciences 


\section{INTRODUCTION}

The book presented to the reader differs from all publications on the history of the USSR nuclear weapons (NW) development in two respects.

First, it is entirely devoted to fundamental research on various theoretic physics sections conducted by NW developing scientists during their efforts on weapons at Sarov (Arzamas-16) Nuclear Center. It is this that the title of the book "In the Intermissions..." stems from. What is meant by the three dots following the word "intermissions"? For most scientists they imply nuclear testing of the nuclear devices developed by them. For some of them this is continuation of their earlier research, getting rid of the scientific problems which have tormented them or a sound way out of their failures in their primary effort. The content and meaning of the "fundamental science" are elucidated in the remarkable essay by Ya.B. Zel'dovich ${ }^{a}$.

Second, it is a collection of papers which were already published earlier in various scientific journals. Being united by a single book cover, the collected fundamental studies enable to recover the creative environment of the theoretical physicists involved in NW development, realize the roots of the methodical approaches relating to their primary activities.

The introduction gives some little known details of the life of Soviet scientists in classified KB-11 (Design Bureau No.11) surrounded with barbed wire, presently known as RFNC-VNIIEF ${ }^{b}$ in Arzamas-16 city $^{c}$.

From the earliest years of existence of the scientists' community here, in parallel with the super-security efforts on development of the nuclear and thermonuclear bomb, papers on fundamental problems of theoretic physics and mathematics began to appear in unclassified scientific journals. The comments of individual papers from their whole set during 50 years of existence of the Arzamas-16 Scientific Center make the second part of the introduction content.

Now we revert to the events which began in June 1946 at the Russia European part center, at the Sarov Cloister site, where monks used to live at 26000 hectares of land granted to them by Empress Yelizaveta in 1743. The Sarov Cloister glory owes to Father Seraphim (1759-1833), a hermit and recluse, whose asceticism, ascetic life and firmness of spirit made the Sarov land with its springs of "holy" water famous. Through the thankful descendants' memory of Seraphim Sarovsky and efforts of monks, by the early 20-th century the Sarov Cloister had become one of principal Russia's sacred places.

\footnotetext{
a The first paper of the book.

${ }^{b}$ The full name: Russian Federal Nuclear Center - All-Russian Scientific Research Institute of Experimental Physics.

${ }^{c}$ From 1996 - Sarov city.
} 
In 1917 the Revolution broke out. The monks disappeared. Stones only remained in buildings, deserted churches, bell towers which fell silent. Since that time these have been used for various purposes and some of them even disappeared at all. Time elapsed, and in 1992 priests returned to one of the old churches, parishioners appeared. In 1997 the first bells restored at the Sarov tower rang anew. However, that time this was at the area of Russian Federal Nuclear Center...

Its foundation began with Governmental Resolution.

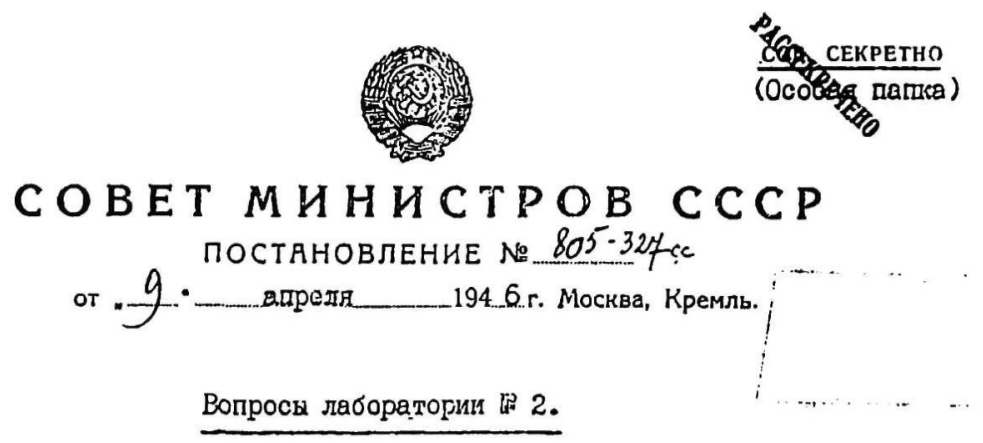

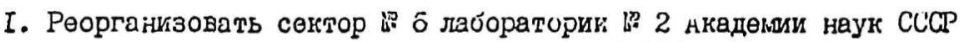

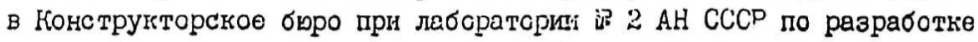
конструкции и изготовлению опытџбх обраэІ:ОВ реагтивнбх двигателей.

2. Указанное. Конструкторское біро впједь иценовать Конструктор-

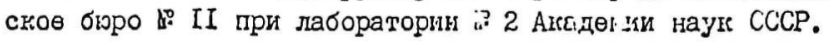

3. Назначить:

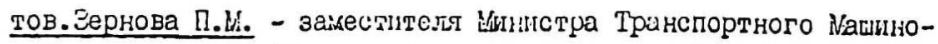
строения Начальником КБ-II с осгобол̈деніси от текудей работы по 1/инистөрству;

просіессора Харитона конструированию и изготовленць стытыше рсзитнвишх пвигателөй. 
TOP SECRET

(Security Dossier)

Declassified

\section{USSR COUNCIL OF MINISTERS RESOLUTION No.805-327ss}

Of 9 April 1946. Moscow, the Kremlin

Issues of Laboratory No.2

1. Reorganize USSR Academy of Sciences Laboratory No.2 Sector No.6 to Design Bureau of USSR Academy of Sciences Laboratory No.2 for jet rocket motor designing and prototype manufacture.

2. Hereafter refer to the above-mentioned Design Bureau as USSR Academy of Sciences Laboratory No.2 Design Bureau No.11 (KB-11).

3. Appoint: comrade Zernov P.M., Deputy Minister of Transport Machine Engineering, as KB-11 Chief, with release from current duties for Ministry; Professor Khariton Yu.B. as KB-11 Chief Designer for jet motor designing and prototype manufacture.

The uranium project works were conducted in a situation of complete secrecy at all levels, including the level of highest-ranking officials ${ }^{d}$. This was vigilantly kept an eye on by Lavrenti Beriya agency. It is this person who was entrusted with arrangement of a completely new and grand affair of the nuclear weapons development in the USSR. The project scientific leadership was carried out by Igor' Vasilyevich Kurchatov.

To find the KB-11 site itself was entrusted to P.M. Zernov ${ }^{e}$ and Yu.B. Khariton. The atomic bomb development effort character required explosive tests involving a large quantity of ordinary explosive. "It was not an easy matter to find an area isolated from population, not far from Moscow, suitable for this kind of work", - recollected Yuli Borisovich.- "We went much over plants which had produced weapons during the War. Well, after long search, on April 2, 1946, P.M. Zernov and myself arrived at a small settlement of Sarov

\footnotetext{
${ }^{d}$ This is testified for by the item where the Laboratory for nuclear device development was disguised by the name of "Rocket Motor Developing Laboratory", which then was considered less secret, even for Council of Ministers members.

eP.M. Zernov - General, the first facility Director. He made a layout of the production buildings and the future city. The majority of his plans were implemented later on.
} 
where once upon a time Holy Seraphim was saying his prays. There was a small plant which fabricated projectiles for "Katyusha's" and other weapons during the War. Around there were impenetrable forest massifs. This made conditions for necessary tests as there was much room well isolated from settlements... Igor' Vasilyevich Kurchatov approved the choice of site. And very extensive work on laboratory foundation and staffing began. Shchelkin ${ }^{f}$ and myself made a strawman of the list of scientists. These numbered 70 . This seemed a large number, well, why so many? None realized the scale of the works then... The first laboratory was situated in the cloister wing. It was necessary to work and make experiments..."

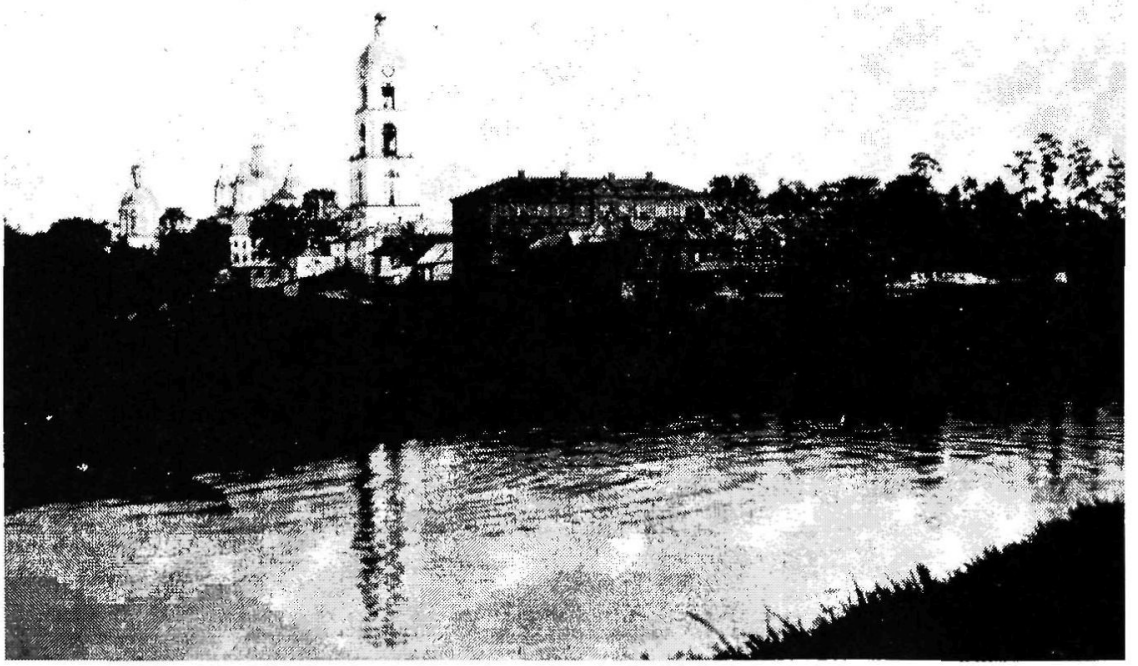

\section{View of the Sarov Cloister in the 30's}

In 1948, on initiative of Yu.B. Khariton and Ya.B. Zel'dovich, a theoretic department was founded at the facility which united both scientists who had already worked on the problem elsewhere and those involved anew in the effort. The Theoretic Department was established to solve fundamental physical problems relating to the nuclear weapons development. Yakov Zel'dovich was appointed the leader of the Department. He was 34 years old. D.A. Frank-

${ }^{f}$ K.I. Shchelkin was a chemical physics scientists, USSR Academy of Sciences Corresponding Member. 
Kamenetsky, N.A. Dmitriyev, G.M. Gandelman, and V.Yu. Gavrilov, Zel'dovich's colleagues by Institute of Chemical Physics, were among the first employees of the Department. He also sought to invite E.I. Zababakhin and E.A. Negin who had graduated from Zhukovsky Air Force Engineering Academy and earlier pursued studying chemical explosive implosion.

Yu.B. Khariton and Ya.B. Zel'dovich began to study chain nuclear reactions as early as 1939 when working at Institute of Chemical Physics. This story relates to I.Ya. Pomeranchuk (Chuk as he was called by his friends, after Landau). According to Zel'dovich, this is as follows: "I remember bus No.13, in the crowd Chuk was narrating about a paper by Perrin. In author's opinion, volcano eruption is a consequence of natural nuclear explosions (uranium fission) deep in the Earth. I rushed to a library and the next day came up to Yu.B. Khariton, and we undertook detailed analysis of the issue with enthusiasm. Our papers appeared in the journal "Zhurnal Eksperimental'noi i Teoreticheskoi Fiziki", a contact with Kurchatov and the laboratory headed by him was established, all of this was a prerequisite of the primary affair of the next twenty years. Well, this was started by a brief narration by Chuk".

Another KB-11 theoretic department oriented to solution of the thermonuclear explosion problem was founded in 1950. It was headed by 55-year-old USSR Academy of Sciences Corresponding Member I.E. Tamm. The skeleton of the team had formed earlier at Lebedev Physics Institute being composed of A.D. Sakharov, Yu.A. Romanov, V.L. Ginzburg, E.S. Fradkin, and C.Z. Belen'ky. Only the first two persons worked at the facility, the others conducted security investigations in Moscow. Note, that the works on the problem were started as early as 1946 at Institute of Chemical Physics when I.I. Gurevich, I.Ya. Pomeranchuk, Yu.B. Khariton and Ya.B. Zel'dovich proposed to use the deuterium fusion reaction for making thermonuclear detonation.

At the initial stage of the uranium project the theoretic works were conducted not only at classified laboratories. Now and then some well-known physicists were involved in collaboration, although were not completely accessible to classified information. For example, L.D. Landau and his disciple I.M. Khalatnikov developed an approximate theory for estimation explosion energy of the atomic and first thermonuclear bombs by a special assignment. But later on the classified theoretic works were conducted without their involvement. Nevertheless, some employees from the KB-11 theoretic department, primarily Ya.B. Zel'dovich, visited the well-known L.D. Landau's workshop at Institute of Physical Problems whenever they could.

In 1949 the mathematical department headed by N.N. Bogolyubov, 40year-old USSR Academy of Sciences Corresponding Member, was established at the facility. V.N. Klimov, D.V. Shirkov, and D.N. Zubarev, his young 
colleagues were invited to work at the department who were entrusted by N.N. Bogolyubov to develop approximate methods for solving nuclear and neutron kinetics problems. Much work on development of computational methods was conducted by V.S. Vladimirov.

Before arrival of N.N. Bogolyubov at the facility the major computational burden was on Steklov Mathematics Institute Division headed by M.V. Keldysh (who later on became USSR Academy of Sciences President). The work of his employees A.N. Tikhonov, A.A. Samarsky, S.K. Godunov, I.M. Gelfand, and K.A. Semendyayev was entirely oriented to needs of the facility. To solve complex applied mathematics problems, teams from other institutes were involved, for example, from Institute of Physics Problems, where the works were headed by N.S. Meiman, from Leningrad Mathematics Institute Devision where studies were headed by L.V. Kantorovich (later Nobel prize winner).

For a person relating to theoretic or computational physics mentioning these names is sufficient to realize what forces were at disposal of the USSR to attack conceptual and practical nuclear and thermonuclear weapons problems.

To collect together a small quantity of prominent talented persons was quite in the spirit of that time. Here is what P.L. Kapitsa (later Nobel prize winner) said regarding this subject: "Though let the science path be predetermined, but the movement along this path is only provided by efforts of a very small number of exceptionally talented people... Therefore, the Institute core can be undoubtedly formed of a small team of very carefully selected scientists".

On their arrival at the facility many of these people got into a situation which was not used for them relating to observance of the secrecy regime. In the early years the bulk of the employees were not allowed to leave the facility. Many people parted their families leaving for a completely unknown site or, as this was said then, to a long-term business trip.

The collective effort on atomic weapons at the facility was of a peculiar form: fellows who worked at different laboratories, departments were not aware of business of one another, did not have right to speak about their job.

At first such strong measures in business communication also touched upon theoretic physicists, mainly, young employees. However, with time a normal business atmosphere established among them. Quite soon the range of problems openly discussed and argued by the theorists expanded beyond the frames of narrow-special, business issues. In some incomprehensible manner it turned out possible to retain inoffensive intellectual habits: Thus, "...David

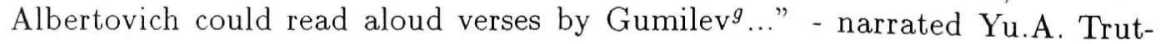

${ }^{g}$ N. Gumilev - Russian poet whose works were forbidden by the USSR state censorship. He was executed by shooting in 1922 as a counter-revolutionary by the All-Union Emergency 
$\mathrm{nev}^{h}$, then a young colleague of D.A. Frank-Kamenetsky working in the same room with him. Or... with an entire revelation and extreme maximalism in judgments inherent in the youth he could criticize T.D. Lysenko ${ }^{i}$ calling him an ignorant and dangerous human being, with it being nowhere else but at the Staff Clearance Commission. This happened to Lev Altshuler ${ }^{j}$ in the early 1950 s.

"...Naturally, the Commission ordered to remove Altshuler", recollected Yu.B. Khariton. "Zel'dovich and Sakharov called on me, narrated about the Commission. I called Beriya, he asked: Do you need him very much?" "Yes, I do," - I answered. "Well, let him stay", Beriya said reluctantly, as it seemed to me".

Why, despite of such circumstances, many Arzamas-16 physicists took a great interest in policy? "For two reasons", - Nikolai Alexandrovich Dmitriyev, a theorist and mathematician, said - "Atomic weapons and especially the hydrogen bomb have altered the situation in the world, and we are involved in this... In particular, the following conversation with Sakharov took place, he said, what your job will be after the hydrogen bomb. Maybe, energy transfer to far distances... However, we came to a conclusion that the physical principles which were good for weapons would not be profitable in the ordinary industry... All of a sudden we understood that on a breakthrough in the nuclear physics no less important problem appeared, i.e., disarmament. At first physics determined policy and then it was necessary to solve political problems with political means. It was this that was responsible for the Sakharov's choice, in particular."

Realization of this came later. But at first and first and foremost there was the exciting work on the major thing, i.e., nuclear weapons development. From appearance of a new idea to its implementation in metal a year or two elapsed, and sometimes this duration contracted to a few months. It would seem that merely no time could be left for any "outside" ideas. However, they do appear and increase in number.

Now let us have a look at business documents and rough notebooks ${ }^{k}$ of Ya.B. Zel'dovich of those times. Compare the dates only on two of them:

The first page of the Ya.B.'s notebook where he began his familiarization with the

Commission sentence.

${ }^{h}$ Yu.A. Trutnev - Russia Academy of Sciences Academician. On recommendation by Sakharov and Zel'dovich he became Theoretic Department Head in 1964.

${ }^{i}$ T.D. Lysenko - Academician, then a formal symbol of success and achievements of the Party and Government policy in the area of agriculture and biology science development.

${ }^{3}$ L.A. Altshuler - a gas dynamics physicist. His fellows by science used to call him "dynamiter Levka".

${ }^{k}$ This notebook is kept in Yu.A. Trutnev's personal archive. 
January 6,1954

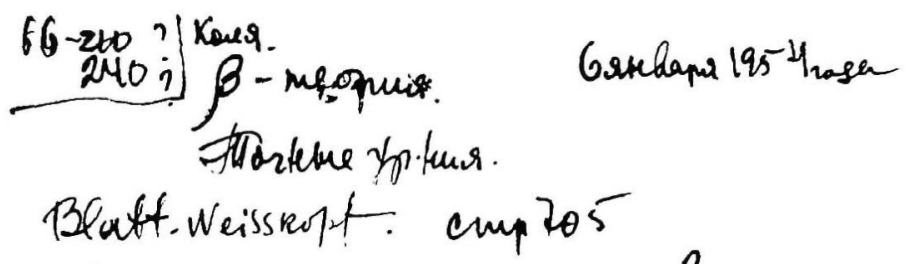

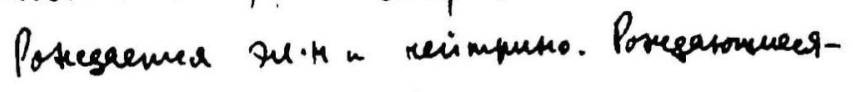
riekertias coemaltum - co zbrgor.

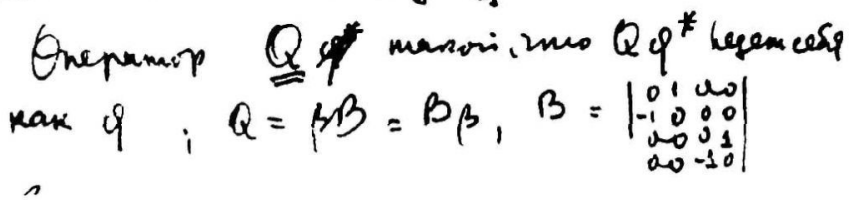

$\beta$-decay theory. And in 20 pages the main directions of the search which terminated with famous paper by him and S. Gershtein on vector current conservation are already clearly formulated

January 14,1954
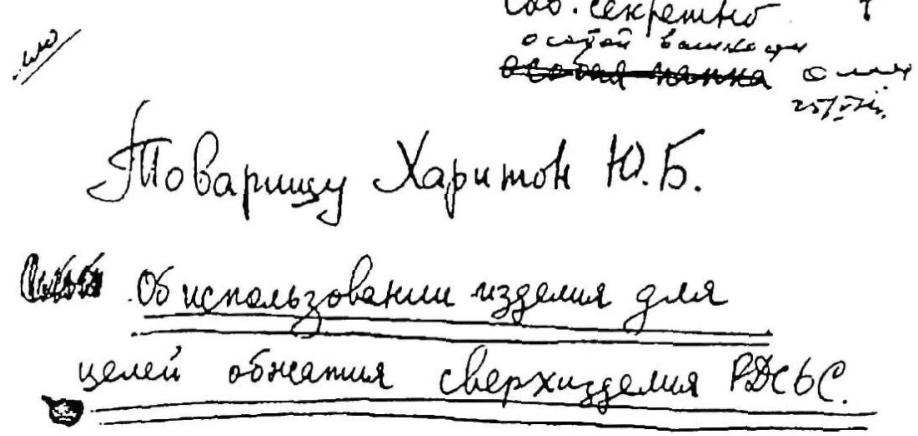

First page of January 14, 1954 report by Zel'dovich and Sakharov. The text reads: "Top Secret. Special dossier. To Comrade Khariton Yu.B. About using the gadget [atomic bomb] for implosion of the supergadget RDS-6s." This report contains schematic of two-stage thermonuclear charge and estimates its performance ${ }^{l}$.

\footnotetext{
'More detailed about this document - see in G.A. Goncharov article (Physics Today, November 1996, Special issue).
} 
The first breakthrough in the secrecy regime was declassification of papers on controlled thermonuclear fusion: the famous presentation by I.V. Kurchatov in Harwell (1956). He was the first to show the scale and significance of the results for the fundamental physics problems which were obtained in the course of the works on nuclear weapons development.

However, both before and many years after this event the technology of appearance of scientific publications by employees from Nuclear Center in "open" publications remained invariable. First the author was said to fill out the document which Zel'dovich called as "oath and pray". In it the author "vowed" in the face of the law that in his work he "...does not betray", "...does not disclose" secret information. Then several commissions had to confirm that the author's materials "do not contain...", they "...do not weaken defensive capability..." and so on and so forth. The last proof was that papers on a similar subject had already appeared in the "open" press. Eventually the author managed to get the desired conclusion regarding possibility of his paper publication.

However, a permanent "violator" of the secrecy regime was ...mathematics. For example, the original problem formulation in the paper by R. Barenblatt and Ya. Zel'dovich arose immediately in the course of classified studies of thermal wave front structure, i.e., structure of powerful radiation pulses at their propagation across condensed medium. And then the universal mathematics language made a "small miracle": then secret problem was re-formulated almost word for word (mathematically isomorphously) in terms of the filtration theory. And on pages of an open journal it became accessible to discussion by other scientists.

Also, there were instances when, bypassing the whole system of the most rigorous "facility" guard, an "outside" person got into the facility area. Such a thing happened to Olga Alexandrovna Ladyzhenskaya, a mathematician, in winter 1961 .

The whole event began when physicists invited her to make a presentation at then general Branch of Physics and Mathematics Science Academy in Moscow. The presentation was devoted to the results obtained by her on the Navier-Stokes equations (on global unique solvability of 2D hydrodynamics problems). After the presentation Yuli Borisovich came up to her, met her and asked her to speak to his colleagues. The proposal was accepted.

The unusual trip stuck in Olga Alexandrovna's memory:

"The visit of the "facility" seemed to me a perfect exotic event. Without any admission, I read no papers and signed nothing. I was brought in and out in about a week with "closed eyes"... I did not even suspect that our discussion took place in Andrei Dmitrievich's room, though I had heard a mention of it." 
At the facility Olga Alexandrovna read an entire course of lectures. To theoretic physicists she was invited by Zel'dovich. He even arranged a ski outing about an extraordinarily beautiful forest massif together with theorists. Sure, she also saw the destroyed church.

"I considered it improper to ask Yuli Borisovich questions about the facility or the church. Once they do not say, then they can not. Moreover, I did not want to put anybody into an awkward situation. As late as we returned to Leningrad Vladimir Ivanovich Smirnov ${ }^{m}$ and myself understood that we had had a chance to visit Sarov Hermitage".

Another example of solving secrecy related problems is involvement of A.I. Baz' in the works on nuclear physics.

In 1959 on initiative of Yu.B. Khariton and Yu.S. Zamyatnin ${ }^{n}$ a decision was made to establish in Arzamas-16 a team of theorists specializing in the nuclear physics area. By advice of I.V. Kurchatov A.I. Baz', a talented physicist who had got international recognition for development of the theory of nearthreshold phenomena in quantum mechanics, was invited as the team leader.

Formally, Baz' was not an employee of KB-11. He went there on longterm missions, he had his own room. This status created no formal obstacles for him for foreign travels. KB-11 employees as classified information bearers were not allowed to contact foreigners.

A.I. Baz' indeed proved a scientist very useful for KB-11. During his work there (he stopped visiting KB in the late 1960s) A.I. Baz' brought up about a dozen of talented disciples. Several papers by them are included in the collection.

The air nuclear testing moratorium (the late 1958 - September 1961) little influenced the work of theorists, nevertheless a number of the employees reoriented their pursuits to fundamental studies.

Ya.B. Zel'dovich arranged a workshop whose subject scope covered astrophysics, general theory of relativity, cosmology. Employees who had pursued bombs thus far unusually easily realized internal mysteries of star arrangement. Everything was as if native. However, the question is: what will happen further when star nuclear fuel has been exhausted, energy is not released inside the star and the star loses its energy as radiation to space. In this situation gravitation begins to predominate over material pressure and the star begins to contract. Ya.B. Zel'dovich and A.D. Sakharov were debating at the blackboard with a piece of chalk in hand, and the audience which had grown quiet faced the

\footnotetext{
${ }^{m}$ V.I.Smirnov - a mathematician, USSR Academy of Sciences Academician, author of the well-known "Course of Higher Mathematics".

${ }^{n}$ Yu.S. Zamyatnin - physicist, leader of works on experimental nuclear physics at the facility in 1948-1967 years.
} 
pattern of their view of the star destiny. This is how setting up and formulation of astrophysics problems for all people present started. The same situation took place with discussion of cosmology and general theory of relativity problems.

In September 1961 nuclear testing was resumed. During the years of the moratorium theorists conducted extensive work to analyze tested device designs and determined lines for their further development making an impressive list of tests. Busy times set in which persisted till late 1962. This was a stressed, responsible effort, the fundamental science concerns became of secondary priority, even in those who had devoted nearly all the time to them during the moratorium years.

The 1963 Threshold Test Ban Treaty put nuclear explosions deep under ground. Each of such experiments became more expensive and required much more efforts for its designing, more sophisticated tools for measuring explosion physical parameters.

The range of problems being studied expanded further and new research areas appeared at the facility.

An important event was advent of large computers at the facility. They enabled to make a large step in the device operation simulation. The new powerful computers provided capabilities for more and more detailed scientific analysis of most complicated nuclear and thermonuclear device explosion processes and development of more perfect designs.

Sure, the portion of the facility scientific research was continuously extended and improved which was devoted to studying nuclear explosion effects, in all respects, from effects of various nuclear explosion factors on separate elements of military equipment to finding out planet-scale consequences in a hypothetical situation of global or local nuclear weapons employment.

From the standpoint of a theoretic physicist it gradually became clear that the "plain physics" (as Sakharov used to say) which was open in the heroic period of making the early nuclear and thermonuclear bombs was not "plain" so much.

However, let us revert to the main subject, i.e., the fundamental studies. In 1965 Penzias and Wilson recorded relict radiation. This event provoked a huge excitation among physicists from all over the world. Arzamas-16 theorists were also impressed by this for the "narrow-professional" reason: it was for the first time possible to measure radiation from the "Biggest Explosion" ("Big Bang") which resulted in generation of the Universe.

"I like most of all the relict radiation bringing to us the information about the first instants of the Universe existence"

- this is how Andrei Dmitriyevich Sakharov said about his predilections in the theoretic physics area. In his, very likely, most famous paper he managed 
to "touch on" these first instants. The paper is devoted to the fundamental problem of our Universe arrangement, the issue why in the Universe matter predominates over antimatter or, as physicists use to say, the issue of the Universe baryon asymmetry. If the Universe were symmetric, the quantity of particles in it would be equal to that of antiparticles, at their collision they would be mutually annihilated, and "light" quanta, i.e., photons, would solely exist instead of us and the matter surrounding us. Why did the "rescuing" matter-antimatter asymmetry arise? Within the (then) conventional ideas this was unexplainable.

In his paper Andrei Dmitrievich suggested an idea of proton decay, of instability of the major Universe "brick". The case in point was that the proton was not everlasting and had a finite lifetime which was billions of billions times longer than the Universe lifetime. By the Sakharov's theory it turned out that this very weak effect under the condition of time reversibility violation in microprocessors (CP-violation) could account for why no complete mutual annihilation of matter and antimatter occurred at the early instants of the Universe appearance. And why a small proton residue arose sufficient for generation of all galaxies, stars and planets.

At first this paradoxical conclusion touching upon the very fundamentals of the microworld notions was accepted by scientists with severe distrust. However, in several years (by the mid 1970s) in the theorists' arsenal theories appeared which confirmed the ideas of the paper by Sakharov. The detailed computations of proton decay time $\left(\tau \sim 10^{31}-10^{32}\right.$ years $)$ conducted within the Great Union Theory (GUT) allowed to expect experimental detection of the proton. Moreover, the hopes which appeared, as then it seemed to many scientists, were sufficient to motivate worldwide construction and startup of most complex facilities.

Results of experiments on these facilities are presently known: the proton decay time is longer than that originally estimated within the Great Union Theory. In this connection it is worthy to pay attention to the fact that the "naive" proton decay estimation in the paper by Sakharov itself provides a considerably higher value of $\geq 10^{50}$ years.

In science everything is frequently determined with several key principles. The community of scientists working on solution of a large-scale problem is arranged similarly. Among Arzamas-16 theoretic physicists several key figures are distinguished, primarily Sakharov, Zel'dovich, and Dmitriyev.

Nikolai Alexandrovich Dmitriyev was not awarded any high scientific titles to, did not occupy high-ranking official positions. He was merely one of main theorists at the facility in the period of the nuclear and thermonuclear weapons development. His contribution to this affair proved extremely large. 
Many Arzamas-16 employees narrate that Sakharov used to say often: "It is necessary to ask advice of Kolya".

Nikolai Alexandrovich comments on this as follows:

"In my opinion, this is a legend devised by Zel'dovich. The point is another, that is capability to critically assess any proposal of any person. I do not trust authorities very much. And, probably, it would be useful for somebody to hear my standpoint. That is all."

Nikolai Alexandrovich began his scientific activities as a pure mathematician. He came to the facility in 1948. He himself explains the reason for which he made a decision to pursue a new business as follows:

"When in 1945 the Americans dropped the atomic bombs onto Hiroshima and Nagasaki, I understood that such things were possible and understood that I had to be involved in this. I did know that there would be much blood without the atomic bomb. We had just left the war, horrible and merciless. And after the explosions in Japan it became clear that the next war was possible and real if we did not have the weapons like those of the Americans. So that to me this was a purely political decision".

And once again, reverting to the works on the early nuclear bombs, to the atmosphere of those times, he remarks:

"I can not distinguish anybody. This was a collective effort. Maybe, it is worthy to distinguish solely Khariton. His role in the project as a whole is extremely high. He is an embodiment of responsibility. He treated any problem extremely seriously. The highest trials a human being is capable of fell to Khariton's lot. ...And not only technical. Nothing was neither major nor minor to him - everything was important".

And the question: could another person cope with this? was answered by Nikolai Alexandrovich as follows:

"Probably, he could, but I can not imagine this. At least, among those who I am familiar with or whom I know."

It seems to us that the life history of Yuli Khariton can be inferred from...as few as two photos. 


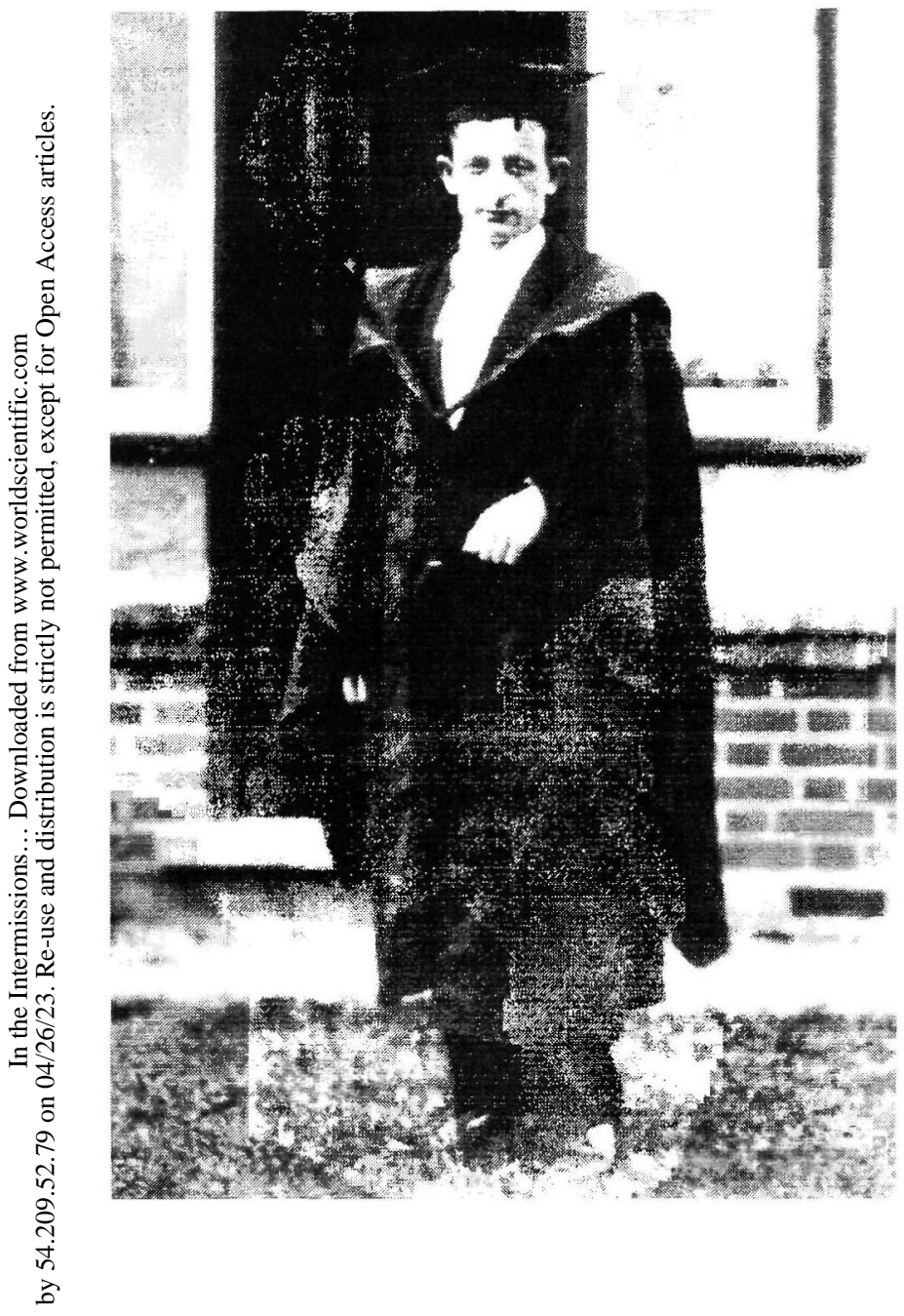

The year 1928. Khariton hosted by Kavendish Laboratory. After passing an examination to Rutherford and Chadwick and conferment of the Philosophy Doctor title. 


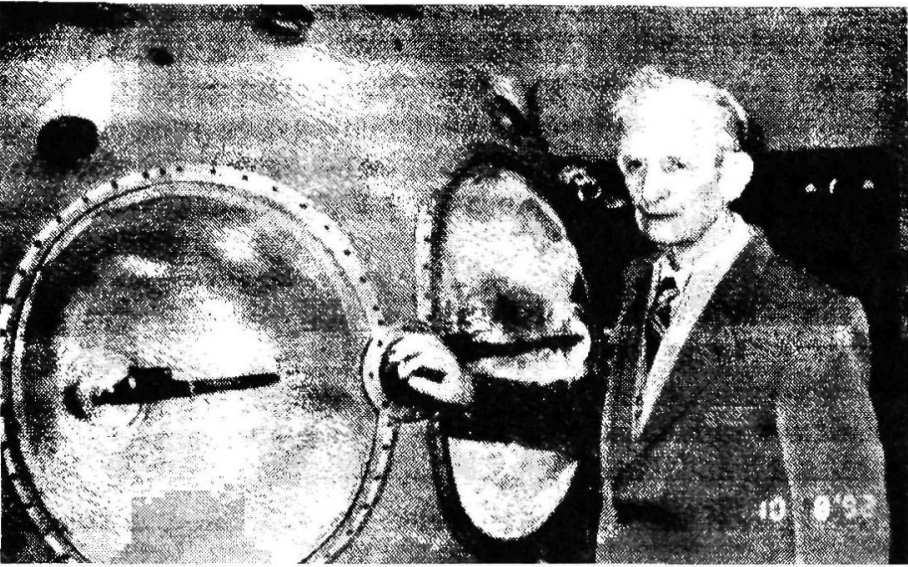

1992. Academician Yuli Khariton in Arzamas-16 Nuclear Weapons Museum near the RDS-1 atomic bomb mockup.

When being in Russia, hosted by physicists at Nuclear Center in the Urals ${ }^{\circ}$, Edward Teller said in one of discussions: "If one meditates upon the past, in it a large role is that of Khariton. Presently I am writing memoirs. They will be called as follows: 'What human eyes saw'. I know that Yuli Khariton is writing memoirs as well. I believe, his memoirs will not be dramatically different from mine". Judging by the results of the effort, these indeed should not be different.

The main Khariton's "manuscript" was written by him in the co- authorship with thousands of people in Russia who developed nuclear weapons and a largest scientific center of the country - Russian Federal Nuclear Center Arzamas-16 - with their talent and labor.

The real nuclear disarmament process is currently underway in the world. How rapid the advance will be and how far the states will go along this direction is yet known to nobody.

For some reasons in the public opinion the conviction arises that a simplest and most efficient method to speed up the process is to reduce allocations as much as possible or abolish scientific centers relating to development of weapons (not only nuclear) whatsoever.

In practice implementation of this receipt is even simpler which leads

oThe second Russia Federal Nuclear Center in the city of Snezhinsk. 
to a consequence very dangerous, in our opinion: to curtailment of works in the fundamental science area. Here, in Russia this is felt most acutely. However, in other countries this trend has clearly showed up as reduction in the governmental allocations for fundamental studies and attempts to entirely transfer such expenses to private corporations. Very many words have been said and written about the need to save money of tax-payers. In the world there is a large quantity of vitally important needs for which funds are permanently insufficient. This is actually so.

However, the truth also is that since the ancient times when people had very limited capabilities and means to maintain life they united three major fundamentals of being: "The God in the heaven, - love and power on the Earth, issues of the Universe in their minds."

As in life of every person, no matter who and what he is, an instant sets in inevitably when he asks himself: "What for am I on this Earth? Whence did I appear and Where will I pass away? Who needs All of this?".

So far nobody succeeded in answering these questions quite satisfactory. Nevertheless, the man do not give up his efforts to approach to the solution of the great mysteries. One of the means for this is the fundamental science.

"The fundamental science is also needed because it satisfies spirital human demands"- Ya. B. Zel'dovich states. This idea is the principal concept in his paper "Social significance of the fundamental science common to all mankind" $p$ which opens our collection.

Following are brief comments on the papers.

I. Astrophysics. Microscopical processes occuring in stellar interiors due to the gravitational squeezing have much in common with those resulting from the implosion in nuclear devices. At the facility, this was first realized by David Frank-Kamenetskiŭ.

His astrophysical studies began in the late 40's. The well-known books by Eddington [1] and Chandrasekhar [2] served him as a source of astrophysical information, and gave impetus to write his own monography. Based on his results obtained by the mid-50's (one of his papers appears in Sec.I), he embarked on his intention. However, in 1956, Frank-Kamenetskil left the facility. The monography [3] was published in the USSR three years later.

At this juncture, the story about the astrophysics stimulated by the nuclear shot science might finish. But a new turn appeared: towards the end of 1958,

${ }^{p}$ Journal "Voprosy Filosofii", No.6, pp. 57-62, 1985. This is the only work in the collection performed by the author when he was no longer an employee of Arzamas-16 RFNC. 
with the advent of the moratorium of nuclear tests, Yakov Zel'dovich was infected with the "astrophysical virus". A little later, this "sickness" goes over to Andrel Sakharov. Further, the epidemic extended to many young theorists. Thus, it sounds like Gospel opening: David bore Yakov, Yakov bore Andrĕ, etc.

Zel'dovich recollected his astrophysical debut appealing to humorous passages of Saltykov-Shchedrin (a Russian writer): "I have found a reference to 'a teacher of calligraphy who, exceeding the bounds of his competence, indulged in cosmography' ...The hint is rather clear because my initial competence is the chemical and theoretical physics, not astronomy."

Zel'dovich's temperament kept him from restricting to individual astrophysical studies. He established his own scientific schoole. In the course of the moratorium, he got the permission for a group of about 15 his disciples to visit regularly Landau's lectures and seminars for improvement of their skill.

The investigation by N. A. Dmitriev and S. A. Kholin presented in Sec.I was initiated at a Landau seminar where the question arose whether neutron stars are tolerant to variations in their basic characteristics. The problem seemed not quite simple which intrigued 24-year-old Serger Kholin who had recently graduated from the Leningrad University. Zel'dovich encouraged his "sporting heat". Kholin joined forces with elder and more experienced colleague N. A. Dmitriev. They together quickly coped with the task. The obtained result gained recognition; it was referred to in the famous Course of Theoretical Physics by Landau and Lifshitz [4].

The question whether the collapse of stars was inevitable was quite topical in the 60's. Zel'dovich burned with desire to clarify the situation. He realized that new constructive approaches were required for the progress; together with his disciple Mikhail Poduretz they were the first to apply the methods of statistical physics in the curved space of General Relativity. Their paper (appearing in Sec.I) is quoted by C. Misner, K. Thorn, and J. Wheeler [5] as an important step along the path to resolving the collapse problem. (Interweaving stories of astrophysical studies in the USSR and the USA at that time are fascinatingly described in Thorn's book [6]).

In early 1959, when consequences of the $V-A$ version of weak interactions were the focus of attention, Bruno Pontecorvo from Dubna noticed that deceleration electron on a nucleus could be due to the neutrino pair emission. If this process plays a considerable role in stellar interiors, then the neutrino radiation carries away some energy, freely penetrating layers of stellar matter. Zel'dovich spoke about this hypothesis at his seminar and suggested volunteers to verify it by accurate calculations. Victor Pinaev, one of Zel'dovich's disciples, took interest in it. His enthusiasm was shared by G. 
M. Gandel'man. They managed to show that the neutrino Bremsstrahlung might dominate over ordinary electromagnetic Bremsstrahlung at the last stages of stellar evolution. Zel'dovich arranged their meeting with Pontecorvo in Moscow. This resulted in Pontecorvo's note [7] and the paper presented in Sec.I. These papers evoked a warm response. Then a flow of research into alternative more effective mechanisms of neutrino emission of stars followed.

The Sakharov's paper on the induced gravitation selected for this book was favorably accepted and received wide recognition in the 80 's when ideas of quantum gravitation became quite tangible within the superstring framework. It was discussed by many eminent reseachers, see e. g., [8]. Zel'dovich had previously hypothesized that the cosmological constant originated from zero oscillations of boson and fermion fields and communicated this to Sakharov. The idea of mutual cancellation of boson and fermion contributions to the cosmological constant was, in Sakharov's view, separated from the discovery of the supersymmetry by a single step. The fact that neither Sakharov nor Zel'dovich guessed this was an annoying Sakharov's reminiscence [9].

On departure of Zel'dovich and Sakharov from the facility, the intensity of fundamental researches decreased. Theorists involved in the dash related to penetrating into fundamentals of the Universe in the 60 's, with leaving their leaders, experienced a drift towards studies directed to the development of new weapons systems. However, when difficult physical questions arose, not only former disciples, but also representatives of new generations of theorists of VNIIEF, unfamiliar personally with Zel'dovich, might appeal to him for advice coming to him in Moscow ${ }^{q}$.

In 1984, M. V. Gorbatenko and A. V. Pushkin derived a simple conformally invariant extension of Einstein equations (this result is presented in Sec.I) which allowed the interpretation in terms of Weyl's geometry. Later on, this led them to conclude the possibility of a certain dependence of particle's quantum numbers on its history in the curved space-time. In their quest to verify their conclusion experimentally by comparing the nuclear spectra of terrestrial matter and that of meteorites, they asked Zel'dovich to support their intention.

The response of Zel'dovich was instantaneous: "I reject the idea of the Pauli principle violation. If the conformal invariance contradicts the identity of particles then let the conformal invariance suffer from this."

Categorical claims were inherent in Zel'dovich. However, he was not a dogmatic person and could change his scientific beliefs even in his maturity. Could anyone say whether he was just adamant in giving his conceptual preferences if once he was still alive at the time of the 'second superstring revolution'

${ }^{q}$ Personal contacts with Sakharov were very occasional 
and owerwhelming pressure of the idea of duality? However, it is not impossible that overcoming difficulties associated with the dilaton in the superstring theory will abolish the conflict between the geometrical view of the conformal world and the quantum identity requirement altogether.

Search for an answer to the question led Pushkin to a theoretic scheme relating to a unique mathematical item, i.e. simple finite group "MONSTER". Some key points of the scheme are presented in a note by Pushkin which terminates the section Astrophysics.

II. Particles and fields. The papers of Pomeranchuk and Bogoliubov presented in Sec.II reveal the hand of either.

Pomeranchuk attached particular significance to asymptotical dependences and let parameters be tending to zero or infinity even where their finite values were usually considered. This was a novelty for theorists at the facility.

The usefulness of the asymptotic estimations also showed up in the paper by Fermi [10] which gave an impetus to writing the presented paper by Pomeranchuk. Fermi had proposed a statistical description of cosmic showers. When specifying this description, in his paper Pomeranchuk properly estimated the size of the region where the multiple particle generation occurs and made modifications to the Fermi theory. This is the first paper from the paper series by Pomeranchuk on the theory of strong interactions at asymptotically high energies where he achieved most prominent results.

The paper by Bogoliubov is the next to last in the series of investigations signifying the advent of a new line in quantum field theory which is now spoken of as the axiomatic scheme of Bogoliubov. It was already outlined in that paper. A fundamental element is taken to be $S$ matrix, a functional of classical fields $g(x)$, which are treated as functions of switching the interaction. It is just the paper that the reason for ultraviolet divergences of matrix elements of $S$ matrix is explicitly indicated: the product of distributions is ill-defined mathematically.

A relation between local operators and the variational derivatives $\delta S / \delta g(x)$ is already traced clearly. It seems that everything is prepared, and it only remains to take the last step to formulate the micro-causality condition, in order that $S$ matrix be entirely determined solely by the requirements of covariance, unitarity and micro-causality, without resort to Hamilton dynamics. But this step [11] was fated to take only two years after Bogoliubov's departure from the facility.

When reading the Bogoliubov's paper, one experiences a surprising feeling that he, being present on the eve of a scientific discovery, knows that it has been already made. Such a "superposition of states" of expected and accomplished 
miracles is typical of his creative work altogether. It might probably be more clear in the light of his joky reminiscences of his childhood: "If I was told a fairy tale involving $B a b a Y a g a^{r}$, I agreed to believe in her abilities to work wonders, but I called for these abilities to be preserved invariant over the course of the tale".

Further, Sec.II presents the L. V. Ovsyannikov's paper where the renormalization group equations are converted to the form most amenable to the analysis of field models, and their exact solutions are found. N. N. Bogoliubov and D. V. Shirkov [12] suggested them to be referred to as the Ovsyannikov equations.

The story of writing of the paper is as follows. Lev Ovsyannikov, then already mature scientist, was present at testing some gadget. His younger colleague Dmitriı Shirkov also arrived at the test site. The test was delayed due to unfavorable meteorological conditions. Physicists were bored, played cards, and talked. Note that, at the Semipalatinsk test site, a 'dry law' was severely observed that time. One languishing day, Ovsyannikov asked Shirkov to speak about some interesting problem. Shirkov had already partially resigned in KB-11 and, spent much time in Moscow, where, together with his supervisor Bogoliubov, he developed the idea of the renormalization group, which had recently arisen, in parallel with writing their monography [12] which presently has become one of the most renowned books on quantum field theory.

It was possible for a while to be distracted from cares regarding the test, and the theorists were absorbed in the world of mathematical abstractions. After the discussion, Ovsyannikov was in the state of deep thoughtfulness, and soon disappeared from the company. Next day, Shirkov saw the manuscript of the prepared paper where the problem outlined by him was quite elegantly solved. Two names were written on the title-page: Ovsyannikov and Shirkov. Shirkov made a protest against his co-authorship. He considered his desert to be mere providing a useful guide to gaining insight into the problem. This, in his opinion, might be, at best, acknowledged at the end of the paper. The paper was published under the only name.

The paper of Ya. B. Zel'dovich and S. S. Gershtein involved in Sec.II is remarkable in that it was the first to indicate that the coupling constant of the vector variant of universal weak interaction is unaffected by strong interaction effects, much as the electric charge of proton remains fixed. Such a statement was of only academical interest at that time since the experiment in the early 50 's strengthened the case for the scalar-tensor coupling. Because of this, the authors made the reservation: 'It is of no practical importance, but very interesting methodologically ...' The observed analogy between the vector

${ }^{r}$ Enchantress somewhat similar to Kalypso 
variant of weak interactions and the electromagnetic interactions became of fundamental importance upon discovery of the $V-A$ weak interaction theory. The idea of the vector current conservation was crucial in the development of the gauge field theory and the discovery of the unified nature of weak and electromagnetic interactions. It stimulated the advancement of such fruitful lines in the particle physics as the partial conservation of axial current, current algebra, and vector dominance.

The paper by Sakharov on the baryon asymmetry of the Universe (as well as his paper on the induced gravitation) is a top of his creative work. Sakharov considered this paper to be the best of all accomplished by him in theoretical physics. Ideas of the paper gained recognition in the middle 70's when eyes were fixed on the proton instability, one of few indirect consequences of the grand unification theory that seemed to be open to its verification by available experimental facilities. It is curious that every idea concerning the baryon asymmetry crossed his mind during the preparation of a popular note on a similar subject. An immediate push was the observation of S. Okubo that a consequence of $C P$ breaking might be a difference between partial decay widths by certain channels for a given particle and antiparticle, with coincident total probabilities. An off-print of his paper presented to E. L. Feinberg was furnished with the epigraph ${ }^{s}$

From S. Okubo's effect

Stems a high-temperature fact:

A crooked figure's fitted coat

Has to the Universe been sewed.

The paper by Kosyakov which terminates Section II is the first one openly published by a VNIIEF employee in an international journal. The paper gives a non-trivial solution to the classic Yang-Mills equations with a source composed of two colored point particles.

III. Nuclei and neutrons. The papers by Zel'dovich, Shirkov, and Vladimirov presented in Sec.III are associated with brilliant pages of the history of physical discoveries at an early stage of the existence of the facility.

The paper on cold neutrons by Zel'dovich demonstates his typical approach to scientific problems that was in splitting a challenging task into several more simple problems amenable to solving by physically transparent methods with not too sophisticated mathematics. This did not mean that he disregarded the apparatus of contemporary theoretical physics. The point is that resert to the physical intuition was more natural to him; it enabled the pathway from initial ideas to the experimental verification of final theoretical results to be globally

${ }^{3}$ Translated by B. P. Kosyakov 
minimimized. Among prominent physicists of the 20th century, Fermi worked in a similar manner.

The idea of the muon catalysis was first advanced by A. D. Sakharov in 1948 , even before he was engaged in research on fusion problems ${ }^{t}$. In a report of the Lebedev Physical Institute (that was declassified as late as 1990), he gave a pattern of formation of mesoions of deuterium and estimated the probability of the nuclear tunneling responsible for the fusion reaction. Zel'dovich came to this idea independently in 1954, and, in a conversation with Sakharov, became aware of the existence of the report. In his paper, Zel'dovich refined the estimations and indicated new mechanisms of the muon catalysis. Among those, as became clear at a later time, the most important was the mechanism of a resonance enhancement of the rate of the mesomolecule formation provided that a weakly bound state is available. Scientists of 50 laboratories from fifteen countries studied the muon catalysis in the 90 's.

Three papers devoted to the problem of the neutron transport are presented in Sec.III. The first two consider approximate solutions to the stationary kinetic equation; they were carried out by D. V. Shirkov and V. S. Vladimirov in the late 40's and early 50's. The third was written by S. A. Kholin about 15 years later; a solution to the nonstationary kinetic equation was found in it. Each attracted much attention in the literature, moreover, the Vladimirov method proved a complete constituent of the modern computational approach to nuclear reactors. It is interesting that the Vladimirov's method (developed in the pre-computer era) is obviously directed to the optimization of succeding algorithms of numerical computations. Unlike the method of B. Carlson developed in Los Alamos about the same time, the Vladimirov's method can be readily extended to multidimensional problems without loss of accuracy and with preservation of the stability of numerical computations.

In the 60 's the VNIIEF research activities on theoretical nuclear physics were related to the formation of the Baz' school. Insight into its works is provided by the paper by Baz' and Zommer appearing in Sec. III which assesses the effect of near-threshold phenomena on nuclear reaction cross-sections.

Anatoliı Tyapin, a member of the Baz' team, missioned to the Kurchatov Institute, met there with V. M. Strutinskil. This meeting determined their further scientific fortunes. Their paper presented in Sec.III became a precursor of the droplet model and gave impetus to developing the shell-correction method. Both approaches are now well known and widely used in nuclear physics. In particular, the shell-correction method generated the concept of the two-

\footnotetext{
${ }^{t}$ Sakharov suspected that this investigation had served as an argument in favour of his incorporation into the Tamm's team specially established for studies on the thermonuclear weapons [9].
} 
bump barrier fission. The paper by Tyapin provides theoretical fundamentals of the method.

Note that many studies were not directly related to weapons demands. Baz' and his disciple Vladimir Rybachenko embarked on an analysis of the notion of time in quantum mechanics. Time is known not to be an operator in quantum mechanics. However, as it was shown by Baz' [13], it is possible to determine the operator of the length of time it takes a particle to stay in a given volume. The paper by Rybachenko presented in Section II shows how this approach can be used when the particle passes a potential barrier region. Also, the paper had a practical motivation associated with estimation of nonresonance type nuclear reaction duration.

The paper by Startsev relates to more recent studies on the nucler physics theory. He managed to combine his method of hydrodynamical description of the detachment of the liquid drop from the capillary edge in the gravitational field with the ideology of the model of nuclear liquid [14] to give a beautiful description of deformations of an atomic nucleus up to its fission.

IV. Fundamental and applied. Needless to say, the bulk of physical investigations at VNIIEF was always directed to applications. A less evident fact is that widely separated studies - from applied ones embodied in 'metal' to the essentially abstract - were often carried out by the same people. The mere specificity of research related to nuclear weapons kept the scientists from closing within a narrow range of their favourite interests.

The book by Ya. B. Zel'dovich and Yu. P. Raizer "Physics of Shock Waves and High-Temperature Hydrodynamic Phenomena" provides some insight into the applied research at VNIIEF and the progress achieved during the initial two decades. Results in the area of physics of extremely high temperatures and densities as well as lines of the attainment of such states are discussed most completely in it. The book is renowned throughout the world. This is the reason why the last section of the present volume considers only few results obtained in the course of applied studies yet interesting from the viewpoint of the foundamentals of physics.

It is worthy to dwell a little bit on the style difference of theoretic research adopted in the academic circles close to Landau school and at the facility which is exemplified by the book by Zel'dovich and Raizer [15]. It attaches quite noticeable attention to numerical estimations of physical quantities as compared to Landau and Lifshits course of theoretical physics. This difference also affected the attitude to the computer which was often considered as a large arithmometer in the academic science. Its role as an integral part of research was realized there as late as the early 70's. However, for the sake of fairness we 
should mention manifestation of some snobbery of certain "secret physicists" in regard to the computer.

Today, it would be difficult to underestimate the significance of the numerical simulation, rather one can go into the opposite extreme. The papers by V. V. Nikiforov and V. P. Piskunov placed in Sec.IV are samples of a subtle balance between mental efforts of the theorist and "computer degrees of freedom". Founders of the thoretical school in Sarov disliked cumbersome expressions and strived to reduce to a simple model catching the essence of the problem. The imprint of such an approach can easily be traced in the papers by Nikiforov and Piskunov. They offer mathematically justified ways of breaking the problem into separate processes on the basis of a deep penetration into the physics of phenomena under study which allows for reducing the effective dimensionality of the problem and then proceeding to its numerical description with the aid of computer.

Studies of the equation of state play a conventionally important role in the physics of extreme states of matter. The spectrum of ápproaches here is rather wide. In this context of interest are investigations where the information on the equation of state is deduced from the "first principles". The papers by N. A. Dmitriev and V. P. Kopyshev included here provide examples of such investigations.

In the paper by Dmitriev, a method for calculation of the cold pressure in a crystal directly with the wave functions of stationary states is suggested. The standard procedure of determination of the pressure, in view of the thermodynamical relationship $\Delta E=p \Delta V$, provides for calculation of energies of close states of the system. These results in severe errors since the energy of the states is high compared to its variations, even at considerable compression of the crystal. Dmitriev managed to avoid this difficulty. He derived an accurate formula for the pressure composed of the kinetic term, and the Coulomb term related to interactions of cells. A remarkable fact is that neither kinetic fluctuations of electrons nor their electromagnetic interactions inside the cells make contribution to the formula. The result of Dmitriev might be recognized classical by every evidence, and merits addressing in statistical physics manuals.

The paper by Kopyshev completes studies of the equation of state of rarefied plasma originated by Anatoliı Larkin who left VNIIEF a little bit earlier and began his successful academical career. To date, the approach used by Larkin and Kopyshev is most substantiated and rigorous.

The paper by M. F. Sarry, a brief version of his review paper published in 1991, involved in Sec.IV, is intimately related to the problem of the equation of state. Sarry suggested a new method for calculation of correlation functions in statistical physics offering a diversity of advantages over the method of the 
two-time Green's functions which became very popular after appearance of the paper by D. N. Zubarev [16]. The Sarry method reduces the correlation functions computation to solution of their algebraic equation system. This can be done with the condition of exact self-consistency for the operator system of the original problem. The Jacobi operator identity is used for this condition.

In the early 70's, much effort was made to construct new high-energy lasers with low light beam divergency. At that time, a phenomenon named the optical phase conjugation [17] was experimentally discovered at the Lebedev Physical Institute [18]. This discovery was given a proper appreciation at VNIIEF where its experimental studies and the inquiry of feasibilities of a high-power laser utility were initiated. Theoretical investigations of the origin of this phenomenon and possible predictions of its new forms began thereat.

Scientific Leader of the Institute Yu. B. Khariton admired with the discovery. Trying to look into possibilities of weapons applications, he posed the question to the leadership of the country as to the suspension of open publications on this subject in the USSR. His initiative was not supported. Nevertheless, the paper by Kochemasov and Nikolaev with pioneering theoretical results obtained in 1973 was submitted to the journal Kvantovaya Elektronika as late as 1975 . For unknown reasons it could not be published earlier than 1977.

The idea of magnetic cumulation was advanced by Sakharov in 1952. Since then, extensive research in the area of high energy densities related to megagauss pulsed magnetic fields has been performed at VNIIEF. The paper by Garanin, Pavlovskiř and Yakubov included in Sec.IV is devoted to the study of a difficult problem of discharge at the surface of insulator which magnetic flux flows through. The authors observed that the discharge area is optically thin, and managed to use this fact to obtain an analytical solution.

Unrealized epic of the "star wars" remained in the past. The agiotage related to the development of the X-ray lasers where the nuclear explosion energy is used for their pumping gave place to a quiet scientific interest in lasers with the soft X-ray emission having no direct weapons applications but probably promising for making holograms of microscopical biological structures. The paper by Gasparyan et al. is devoted to this matter. Equations of kinetics

$\vec{b}$ of processes in plasma where hydrogen-like ion level population is essentially dependent of slow multi-particle colisions of ions are proposed and theoretically investigated in it for the first time.

The work of a theorist at VNIIEF is rather peculiar. The mission of the theorist does not come to the end on constructing a theoretical model of a given phenomenon and coding its numerical description. Then he should take care of optimal ways of the experimental verification of the computed results. 
Thus, one day, the question arose of the verification of the model described in the paper by Golubev, Solov'ev and Terekhin presented in Sec.IV. This could be done best in the setup built at the Institute of Theoretical and Applied Mechanics in Novosibirsk. Director of the Institute was N. N. Yanenko, who, in the far past, had also visited the facility and kept friendship with theorists of VNIIEF. This played a decisive role in the conduct of complex and expensive experiments.

Self-similar solutions have always been of high importance in the theory of extreme states of matter. In the pre-computer era, they were used as a reasonable first approximation for perturbation theory base calculations. With the advent of computers, such solutions gained widespread acceptance for testing numerical codes and robustness verification of difference schemes especially near singularities of solutions to differential or integral equations ${ }^{u}$.

The paper of R. M. Zaidel and K. A. Semendyaev which appears in Sec.IV describes a nontrivial solution for heat a wave converging to the center ${ }^{v}$. A self-similar solution for the focusing shock wave is known to exist. Its motion to the center is unaffected by disturbances generated far behind the front. For the heat wave in a cold medium with a nonlinear heat conduction, similar conditions seem impossible since the process is described by a parabolic equation, and perturbations propagate at an infinite velocity. However, the reasoning becomes invalid near the front where the temperature and, hence, heat conductivity factor vanish. The paper shows the existence of a thermal wave where just these conditions occur.

An example of the analysis of self-similar solutions with results which go far beyond an initially stated utilitarian aim is provided by the paper by G. I. Barenblatt and Ya. B. Zel'dovich presented in Sec.IV. They observed that the stability of self-similar solutions of the second kind did not upset provided that the disturbed solution tends to a solution shifted in phase with respect to the non-disturbed solution as $t \rightarrow \infty$. Thus the definition of stability should take into consideration zero modes. Similar ideas associated with spontaneous symmetry breaking emerged independently in quantum field theory and statistical physics somewhat later, and are known today, respectively, as the Goldstone theorem [20] and the theorem of Bogoliubov $1 / q^{2}$ type singularities [21].

$\checkmark$ Acknowledgements. Editorial Board thanks Viktor Mikhailov and Lev Ryabev, leaders of the Ministry for Atomic Energy, for financial support of the

\footnotetext{
${ }^{u}$ An example of this type of modern studies of self-similar solutions is the paper [19] results of which are related to the present paper by Piskunov

${ }^{v} \mathrm{By}$ this is meant the propagation of powerful radiation flux across matter. The process is of great importance in a thermonuclear explosion and is referred to as heat wave
} 
Project of this book publication. In the course of preparation of this book and discussing possibilities of its publication, the Editorial Board had privileges and pleasure being rendered assistance by many people and followed their advices. We are faithfully grateful to them. The enthusiasm and support of Vakhtang Garsevanishvili, Semen Gershtein, Vitaliŭ Gol'danskĭ̌, Maurice Jacob, Dmitrĭ Shirkov are of particular value.

We are also grateful Nuclear Weapons Museum, Arzamas-16, for the presented photo materials.

[1] A. Eddington, The Internal Constitution of Stars (Cambridge UP, Cambridge, England, 1926).

[2] S. Chandrasekhar, Radiative Transfer (Dover, New York, 1960).

[3] D. Frank-Kamenetskii, Physical Processes in Stellar Interiors (Israel Program for Scientific Translations, Jerusalem, 1962).

[4] L. D. Landau and E. M. Lifshitz, Statistical Physics, 2nd ed. (AddisonWesley, Reading, MA, 1969).

[5] C. Misner, K. Thorne, J. Wheeler, Gravitation (Addison-Wesley, Reading, MA, 1972).

[6] K. Thorne, Black Holes and Time Wards: Einstein's Outrageous Legacy (W. W. Norton, New York, 1994).

[7] B. M. Pontecorvo, JETP 9 (1959) 1148.

[8] A. D. Sakharov, Collected Scientific Works (Marcel Dekker, New York and Basel, 1982).

[9] A. Sakharov, Memoirs (Alfred Knopf, New York, 1990).

[10] E. Fermi, Progr. Theor Phys. 5 (1950) 570.

[11] N. N. Bogoliubov, Izvestiya Academii Nauk, Ser. Fiz. 19 (1955) 237.

[12] N. N. Bogoliubov and D. V. Shirkov, Introduction to the Theory of Quantized Fields (Interscience, New York, 1959).

[13] A. I. Baz', Sov. J. Nucl. Phys., 5 (1967) 161.

[14] A. S. Tyapin, JETP Lett. 30 (1979) 461.

[15] Ya. B. Zel'dovich and Yu. P. Raizer, Physics of Shock Waves and High-

Temperature Hydrodynamic Phenomena (Academic, New York, 1966).

[16] D. N. Zubarev, Sov. Phys. Usp. 3 (1960) 320.

[17] V. V. Shkunov and B. Ya. Zel'dovich, Sci. Amer. 253 (1985) 54.

[18] B. Ya. Zel'dovich et al., JETP Lett. 15 (1972) 109;

O. Yu. Nosach et al., ibid. 16 (1972) 435.

[19] A. A. Lushnikov and V. N. Piskunov, Doklady Akademii Nauk SSSR 231 (1976) 1166 .

[20] J. Goldstone, Nuovo Cimento 19 (1961) 154.

[21] N. N. Bogoliubov, Preprint, JINR D-781, Dubna, 1961. 


\section{CONTENTS}

Social significance of fundamental science common to all mankind Ya.B. Zel'dovich

\section{Gravitation and astrophysics}

Oscillatory stability and auto-oscillations of stars

D.A. Frank-Kamenetskii . . . . . . . . . . . . . . 13

Features of static solutions of the equations of gravitation

N.A. Dmitriev and S.A. Kholin . . . . . . . . . . . 18

The evolution of a system of gravitationally interacting point masses

Ya.B. Zel'dovich and M.A. Podurets

Emission of neutrino pairs by electrons and the role played by it in stars

G.M. Gandel'man and V.S. Pinaev . . . . . . . .

Vacuum quantum fluctuations in curved space and the theory of gravitation

A.D. Sakharov

Dynamics of the affinely connected space and conformally invariant extension of Einstein equations

M.V. Gorbatenko and A.V. Pushkin

"Monstrous moonshine" and physics

\section{A.V. Pushkin . . . . . . . . . . . . . . . .}

On the theory of multiple particle production in a single collision

I.Ya. Pomeranchuk 
Variational equations of quantum field theory

N.N. Bogoliubov

General solution to the renormalization group equations

L.V. Ovsyannikov

Meson corrections in the theory of beta decay

S.S. Gershtein and Ya.B. Zel'dovich

Violation of $C P$-invariance, $C$-asymmetry and baryon asymmetry of the universe

A.D. Sakharov

Time of penetration of a particle through a potential barrier

V.F. Rybachenko

Classical Yang-Mills field generated by two colored point charges B.P. Kosyakov

\section{Nuclear physics}

Reactions induced by $\mu$-mesons in hydrogen

Ya.B. Zel'dovich

Storage of cold neutrons

Ya.B. Zel'dovich

Quasistatic drop model of the nucleus as an approximation to the statistical model

V.M. Strutinsky and A.S. Tyapin

The shell correction method

A.S. Tyapin

Effect of threshold levels on the shape of the photoneutron spectrum

A.I. Baz' and V.P. Zommer 
Effect of finite coherence length on dynamics of nuclear fission A.I. Startsev

Synthetic kernel method for neutron diffusion in hydrogenous media D.V. Shirkov

A numerical solution to the transport equation for the spherically symmetrical geometry

V.S. Vladimirov .

\section{Fluid dynamics. Laser. Statistical physics}

On asymptotical properties of self-similar solutions to the equations of nonstationary filtration of gas

Ya.B. Zel'dovich and G.I. Barenblatt . . . . . . . . 150

Limiting solutions of a nonlinear equation of the parabolic type

R.M. Zaydel and K.A. Semendyaev . . . . . . . . . 155

Computation technique for turbulent mixing in one-dimensional flows

V.V. Nikiforov

Reproduction of the spatial amplitude and phase distributions of a pump beam in stimulated Brillouin scattering

G.G. Kochemasov and V.D. Nikolaev

Collisionless dispersion of an ionized cloud into a homogeneous magnetized plasma

A.I. Golubev, A.A. Solov'ev, and V.A. Terekhin . . . . 177

Stationary discharge accompanying emergence of the magnetic flux through the surface of an insulator

S.F. Garanin, E.S. Pavlovskii, and V.B. Yakubov . . . 187 
Effect of plasma microfields on the gain of hydrogen-like ions with photoresonant pumping

P.D. Gasparyan, V.M. Gerasimov, A.N. Starostin, and A.E. Suvorov . . . . . . . . . . . . . . . . . . . . . . 196

The equation of state of a crystal at zero temperature N.A. Dmitriev . . . . . . . . . . . . . . . . . . 208

Certain exact solutions of the nonstationary kinetic equation without taking retardation into account

S.A. Kholin . . . . . . . . . . . . . . . . 215

Second virial coefficients of a plasma

V.P. Kopyshev . . . . . . . . . . . . . . . . . . 223

A new analytical method of calculating correlation functions in quantum statistical physics

M.F. Sarry . . . . . . . . . . . . . . . . . . . . 231

Coagulation Kinetics of Composite Particles

V.N. Piskunov . . . . . . . . . . . . . . . . . . 241

Brief information on the authors . . . . . . . . . 247 